\title{
Clinical results of stereotactic body radiotherapy for Stage I small-cell lung cancer: a single institutional experience
}

\author{
Yoshiyuki SHIOYAMA ${ }^{1, *}$, Katsumasa NAKAMURA ${ }^{2}$, Tomonari SASAKI ${ }^{2}$, Saiji OHGA ${ }^{2}$, \\ Tadamasa YOSHITAKE ${ }^{1}$, Takeshi NONOSHITA ${ }^{2}$, Kaori ASAI $^{2}$, Koutarou TERASHIMA ${ }^{2}$, \\ Keiji MATSUMOTO ${ }^{2}$, Hideki HIRATA ${ }^{3}$ and Hiroshi HONDA ${ }^{2}$
}

\author{
${ }^{1}$ Department of Heavy Particle Therapy and Radiation Oncology, Graduate School of Medical Sciences, Kyushu \\ University, Maidashi 3-1-1, Higashi-ku, 812-8582, Fukuoka, Japan \\ ${ }^{2}$ Department of Clinical Radiology, Graduate School of Medical Sciences, Kyushu University, Maidashi 3-1-1, Higashi-ku, \\ 812-8582, Fukuoka, Japan \\ ${ }^{3}$ Department of Health Sciences, Graduate School of Medical Sciences, Kyushu University, Maidashi 3-1-1, Higashi-ku, \\ 812-8582, Fukuoka, Japan \\ *Corresponding author. Department of Heavy Particle Therapy and Radiation Oncology, Kyushu University, Maidashi 3-1-1, \\ Higashi-ku, 812-8582, Fukuoka, Japan. Tel: +81-92-642-5695; Fax: +81-92-642-5708; E-mail: shioyama@ radiol.med. \\ kyushu-u.ac.jp
}

(Received 19 March 2012; revised 5 July 2012; accepted 24 July 2012)

\begin{abstract}
The purpose of this study was to evaluate the treatment outcomes of stereotactic body radiotherapy (SBRT) for Stage I small-cell lung cancer (SCLC). From April 2003 to September 2009, a total of eight patients with Stage I SCLC were treated with SBRT in our institution. In all patients, the lung tumors were proven as SCLC pathologically. The patients' ages were 58-84 years (median: 74). The T-stage of the primary tumor was T1a in two, T1b in two and T2a in four patients. Six of the patients were inoperable because of poor cardiac and/or pulmonary function, and two patients refused surgery. SBRT was given using 7-8 noncoplanar beams with 48 Gy in four fractions. Six of the eight patients received 3-4 cycles of chemotherapy using carboplatin (CBDCA) + etoposide (VP-16) or cisplatin (CDDP) + irinotecan (CPT-11). The follow-up period for all patients was 6-60 months (median: 32). Six patients were still alive without any recurrence. One patient died from this disease and one died from another disease. The overall and disease-specific survival rate at three years was $72 \%$ and $86 \%$, respectively. There were no patients with local progression of the lesion targeted by SBRT. Only one patient had nodal recurrence in the mediastinum at 12 months after treatment. The progression-free survival rate was $71 \%$. No Grade 2 or higher SBRT-related toxicities were observed. SBRT plus chemotherapy could be an alternative to surgery with chemotherapy for inoperable patients with Stage I small-cell lung cancer. However, further investigation is needed using a large series of patients.
\end{abstract}

Keywords: stereotactic body radiotherapy; small-cell lung cancer; Stage I

\section{INTRODUCTION}

Small-cell lung cancer (SCLC) represents approximately $20 \%$ of all lung cancer and it is basically characterized by rapid growth and early metastasis [1]. Therefore, thoracic radiotherapy combined with systemic chemotherapy has been typically accepted as the standard treatment for patients with limited disease [2]. For patients with Stage I SCLC among limited-disease, however, surgical resection followed by chemotherapy is recommended in the clinical practice guidelines of the National Comprehensive Cancer Network (NCCN) [3] and also the Japan Lung Cancer Society [4]. Nevertheless, we sometimes experience patients with Stage I SCLC who are medically inoperable because of poor pulmonary function, cardiac disease and/or other co-existing diseases. However, an optimal treatment has not been established for medically inoperable patients with Stage I SCLC. Currently, stereotactic body radiotherapy 
(SBRT) has been widely used as one of the curative treatment options for peripherally-located Stage I non-small cell lung cancer (NSCLC) [5-9]. Given that SCLC is generally more radiosensitive than NSCLC, we hypothesized that SBRT would also be effective for Stage I SCLC.

From 2003, at Kyushu University, we began using SBRT for Stage I SCLC patients who were not candidates for surgery. Here, we retrospectively analyse the treatment outcomes of SBRT for patients with Stage I SCLC in our institution, and evaluate the effectiveness and safety of SBRT for this disease.

\section{MATERIALS AND METHODS}

\section{Patients}

From April 2004 to September 2009, 269 patients with lung tumors were treated with SBRT at Kyushu University Hospital. Of these, eight patients with Stage I SCLC were treated with SBRT. In all patients, pathology of the lung tumors was confirmed as SCLC by transbronchial brushing cytology or CT-guided percutaneous fine needle aspiration cytology. The patients' age was 58-84 (median: 74) yeas. Tumor size range was $15-38 \mathrm{~mm}$ (median: 29) in diameter. The T-Stage of the primary tumor at the time of diagnosis was T1a in two, T1b in two and T2a in four patients. Serum levels of the pro-gastrin releasing peptide (ProGRP) and neuron-specific enolase (NSE) before treatment are shown in Table 1. Normal levels of ProGRP and NSE in our institution are less than $46.0 \mathrm{pg} / \mathrm{ml}$ and $12.0 \mathrm{ng} / \mathrm{ml}$, respectively. All patients received 18F-fluorodeoxyglucose positron emission tomography (FDG-PET) and brain magnetic resonance imaging (MRI) for staging in addition to chest-abdominal CT scan. Six of the patients were inoperable because of poor cardiac and/or pulmonary function, and two patients refused surgery. Patient characteristics are summarized in Table 1. All of the patients provided written informed consent.

\section{Treatment planning for SBRT}

All patients were fixed with a thermoplastic body cast combined with a vacuum pillow, arm and leg support, and a carbon plate (Engineering Systems Co., Matsumoto, Japan). The detail of this body fixation system has been described previously [10]. Treatment planning was performed using the 3D RTP machine (Eclipse 7.1.4). The gross target volume (GTV) was identified on relevant lung setting CT images. The internal target volume (ITV) was created individually according to the internal respiratory motion. The planning target volume (PTV) margin was $5 \mathrm{~mm}$ in all directions. Seven to eight multi-leaf-collimator (MLC)shaped non-coplanar static ports of 4- or 10-MV X-rays were selected to maintain target volume homogeneity within $10 \%$, and to decrease the irradiated lung volume receiving 20 Gy or more (V20) to below $20 \%$.

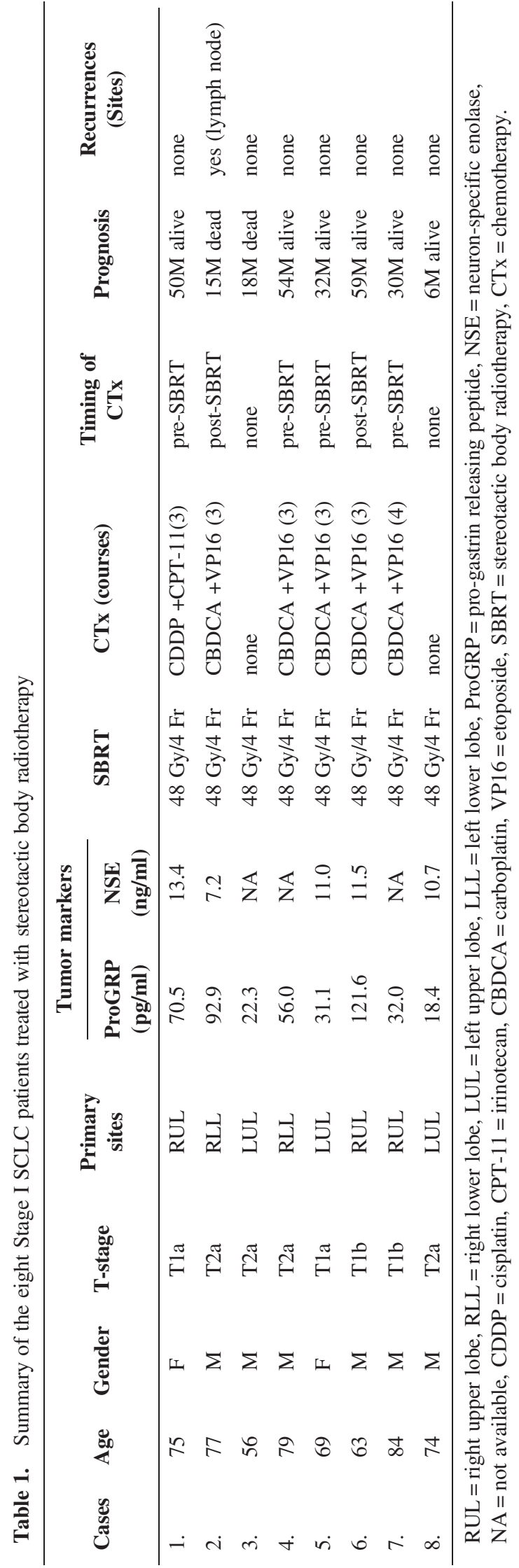




\section{Treatment}

SBRT was given to the PTV with an isocenter dose of 48 Gy in four fractions. Total treatment duration ranged from 4-8 (median =4) days. By using CT and portal images (anterior-posterior and lateral), the isocenter was verified in the first treatment session. In the second and following sessions, portal images (anterior-posterior and lateral) and/or $\mathrm{CT}$ images were used. When respiratory tumor motion was $1 \mathrm{~cm}$ or more, breath-holding irradiation was performed using a visual-feedback-guided breath-holding system developed at our institution $[11,12]$. Chemotherapy using carboplatin (CBDCA) + etoposide (VP-16) or cisplatin $(\mathrm{CDDP})+$ irinotecan (CPT-11) was performed for six patients before or after SBRT. Five of the patients received 3-4 cycles of CBDCA (AUC $=5$ on Day 1$)+$ VP-16 (100 $\mathrm{mg} / \mathrm{m}^{2}$ on Days 1,3 and 5 every 4 weeks), and one received three cycles of CDDP $\left(20 \mathrm{mg} / \mathrm{m}^{2}\right.$ on Days 1,8 and 15$)+$ CPT-11 $\left(60 \mathrm{mg} / \mathrm{m}^{2}\right.$ on Days 1,8 and 15 , every 4 weeks). Two patients with severe cardiac or liver dysfunction were treated with SBRT alone. The details are shown in Table 1. No patients received prophylactic cerebral irradiation (PCI) after completion of SBRT, with or without chemotherapy.

\section{Patient follow-up and evaluation}

After completion of SBRT, patients were evaluated by examinations including chest X-ray and CT every two to three months for two years, and every six months thereafter. Brain MRI and FDG-PET were also performed if needed.

\section{Methods of data analysis}

Survival rates (overall, disease-specific, progression-free), local control rate after SBRT, pattern of failures, and SBRTrelated toxicities were evaluated. Overall, the diseasespecific and progression-free survival rates, and local control rate were estimated with the Kaplan-Meier method. SBRTrelated toxicities were graded according to the Common Toxicity Criteria for Adverse Effect version 3.0 [13].

\section{RESULTS}

The follow-up period for all patients was 6-60 months (median: 32). Six patients were still alive without any recurrence. One patient died from SCLC, and one died from another disease. The overall survival rate and diseasespecific survival rate at three years were $72 \%$ and $86 \%$, respectively (Fig. 1). There were no patients with local progression of the target lesion of SBRT. Only one patient, who was treated with SBRT alone, had nodal recurrence in the mediastinum at 12 months after treatment. This patient refused salvage treatment by radiotherapy or chemotherapy for the recurrence, and received best supportive care in

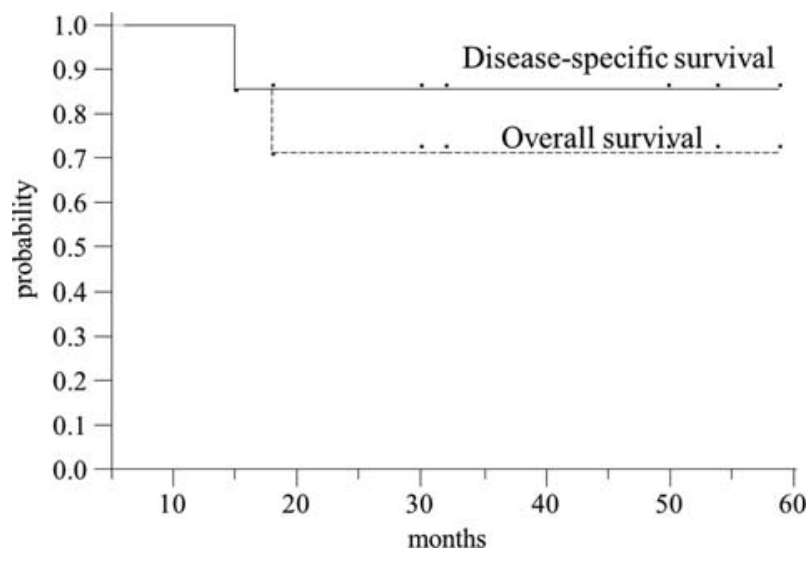

Fig. 1. Overall and disease-specific survival rates for eight patients treated with SBRT for Stage I SCLC.

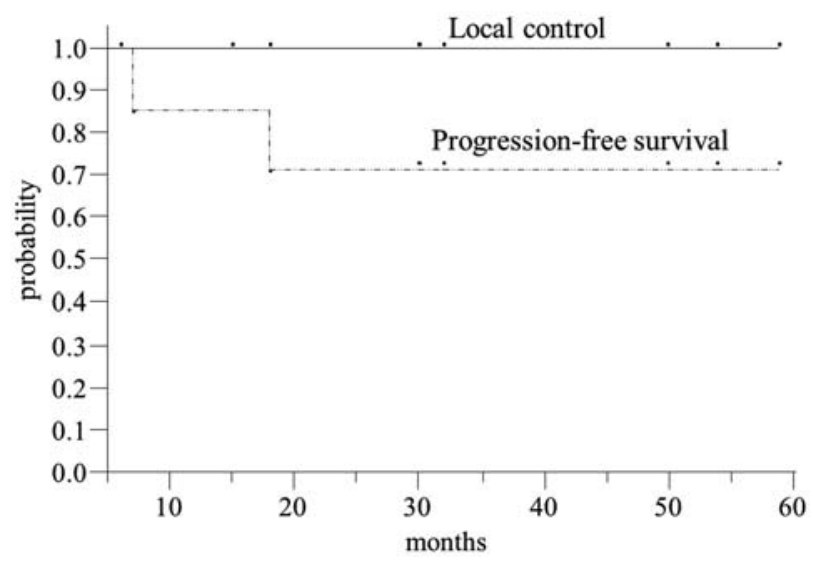

Fig. 2. Progression-free survival rate and local control rate for eight patients treated with SBRT for Stage I SCLC.

another hospital. There were no patients with distant metastasis during the follow-up period. The progression-free survival rate and local control rate at three years were $71 \%$ and $100 \%$, respectively (Fig. 2). No Grade 2 or higher SBRT-related toxicities were observed during follow-up in any patient.

\section{DISCUSSION}

In the present study, favorable outcomes were achieved with SBRT of $48 \mathrm{~Gy}$ in four fractions with or without chemotherapy for patients with clinical Stage I SCLC; the 3 -year survival rate and local control rate were $72 \%$ and $100 \%$, respectively. The treatment was also considered well tolerable because no Grade 2 or greater toxicity was observed. Therefore, SBRT can be a safe and effective treatment option for Stage I SCLC.

Several reports have been published regarding the outcomes of surgery with or without chemotherapy for Stage I 
SCLC. The 5-year overall survival rates after surgery for Stage I SCLC have been reported as approximately 50-70\% [14-17]. Although it is difficult to compare these results directly, mainly because of the difference between the pathological stage and clinical stage, the 3-year survival rate in our study using SBRT compared favorably to those of surgical series previously listed.

In previous reports of SBRT for NSCLC [6] and also metastatic lung tumors [18], favorable outcomes of survival and local control were reported in patients treated with a biologically effective dose, assuming the alpha/beta ratio to be 10 (BED10) over $100 \mathrm{~Gy}$ at the isocenter. In our present study of SBRT for Stage I SCLC, the prescribed dose of $48 \mathrm{~Gy}$ in four fractions (BED10 = 105.6 Gy) at the isocenter was used for all patients, the same as the commonly used SBRT dose for Stage I NSCLC in Japan, because there is no published information regarding the optimal dose of SBRT for SCLC. Complete local control was achieved with no severe toxicity in any patients. Therefore, this prescribed dose is considered sufficient for local control of T1-2a SCLC. However, given that SCLC is known to be more radiosensitive than NSCLC, the total dose of SBRT might be reduced slightly for SCLC. Considering that SCLC is also a rapid growth tumor, more fractionated scheduling may be advantageous in the point of cell cycle effects. Therefore, further investigation is necessary to determine optimal dose and fraction scheduling of SBRT for Stage I SCLC. However, it should be noted that BED based on the linear-quadratic (LQ) model has been shown to overestimate the effect of high fractional doses of radiation, and also not to take reoxygenation as well as cell cycle effect into account [19].

Chemotherapy has an important role in the treatment of SCLC because of its biological behavior, characterized by rapid growth and early dissemination. Generally, a chemotherapy regimen using CDDP (or CBCDA) plus VP-16 is combined with thoracic radiation therapy for limited-disease SCLC (LD-SCLC). Also, adjuvant chemotherapy is generally recommended after surgery for Stage I SCLC in order to reduce the risk of distant metastasis. In the present series, four patients initially received chemotherapy in other hospitals, and then were introduced to our institution because of residual tumors. Two patients received adjuvant chemotherapy after SBRT as planned treatment. In these patients who received neoadjuvant or adjuvant chemotherapy no Grade 2 or higher SBRT-related toxicity was observed. Although the number of patients is too small to draw a conclusion, the sequential combination (neoadjuvant or adjuvant) of chemotherapy may be safe in patients treated with SBRT. In SBRT for Stage I SCLC, therefore, it is better to combine chemotherapy with SBRT. However, the optimal timing of chemotherapy combined with SBRT (neoadjuvant, concurrent, vs adjuvant) is yet to be determined.
Recently, the role of PCI has been established in the treatment of SCLC, especially in LD-SCLC [20, 21]. In our series, no patients suffered from brain metastasis, despite not undergoing PCI. However, the number of patients in this series is too small to discuss whether PCI is essential or not in Stage I SCLC patients.

In conclusion, SBRT plus chemotherapy could be an alternative treatment option to surgery with chemotherapy for patients with Stage I small-cell lung cancer. However, further investigation is needed using a larger number of patients.

\section{ACKNOWLEDGEMENTS}

This study was partially presented at ASTRO 2010, the 52nd Annual Meeting of the American Society for Radiation Oncology.

\section{REFERENCES}

1. Jackman DM, Johnson BE. Small-cell lung cancer. Lancet 2005;366:1385-96.

2. Warde P, Payne D. Does thoracic irradiation improve survival and local control in limited-stage small cell carcinoma of the lung? A meta-analysis. J Clin Oncol 1992;10:890-5.

3. Johnson BE, Crawford J, Downey RJ et al. Small cell lung cancer clinical practice guidelines in oncology. J Natl Compr Canc Netw 2006;4:602-22.

4. The Japan Lung Cancer Society: Japan and Chiba. http:// www.haigan.gr.jp/uploads/photos/261.pdf (20 January 2011, date last accessed, in Japanese).

5. Wulf J, Haedinger U, Oppitz U et al. Stereotactic radiotherapy for primary lung cancer and pulmonary metastases: A noninvasive treatment approach in medically inoperable patients. Int J Radiat Oncol Biol Phys 2004;60:186-96.

6. Onishi H, Araki T, Shirato H et al. Stereotactic hypofractionated high-dose irradiation for stage I nonsmall cell lung carcinoma: Clinical outcomes in 245 subjects in a Japanese multiinstitutional study. Cancer 2004;101:1623-31.

7. Singh D, Yi WS, Brasacchio RA et al. Is there a favorable subset of patients with prostate cancer who develop oligometastases? Int J Radiat Oncol Biol Phys 2004;58:3-10.

8. Nagata Y, Takayama K, Matsuo Y et al. Clinical outcomes of a phase I/II study of 48 Gy of stereotactic body radiotherapy in 4 fractions for primary lung cancer using a stereotactic body frame. Int J Radiat Oncol Biol Phys 2005;63: 1427-31.

9. Zimmermann FB, Geinitz H, Schill S et al. Stereotactic hypofractionated radiation therapy for stage I non-small cell lung cancer. Lung Cancer 2005;48:107-14.

10. Shioyama Y, Nakamura K, Anai S et al. Stereotactic radiotherapy for lung and liver tumors using a body cast system: Setup accuracy and preliminary clinical outcome. Radiat Med 2005;23:407-13.

11. Yoshitake T, Nakamura K, Shioyama Y et al. Breath-hold monitoring and visual feedback for radiotherapy using a 
charge-coupled device camera and a head-mounted display: system development and feasibility. Radiat Med 2008;26: 50-5.

12. Yoshitake T, Shioyama Y, Nakamura K et al. A clinical evaluation of visual feedback-guided breath-hold reproducibility of tumor location. Phys Med Biol 2009;54:7171-82.

13. Cancer Therapy Evaluation Program NCI; Common Terminology Criteria for Adverse events (CTCAE). http:// ctep.cancer.gov/protocolDevelopment/electronic_applications/ docs/ctcaev3.pdf ( 9 August 2006, date last accessed).

14. Davis S, Crino L, Tonato $\mathrm{M}$ et al. A prospective analysis of chemotherapy following surgical resection of clinical stage I-II small-cell lung cancer. Am J Clin Ocol 1993;16:93-5.

15. Rea F, Callegaro D, Favaretto A et al. Long term results of surgery and chemotherapy in small cell lung cancer. Eur $J$ Cardio-thorac Surg 1998;14:398-402.

16. Brock MV, Hooker CM, Syphard JE et al. Surgical resection of limited disease SCLC in the new era of platinum chemotherapy; its time has come. J Thorac Cardiovasc Surg 2005;129:64-72.

17. Tsuchiya R, Suzuki K, Ichinose Y et al. Phase II trial of postoperative adjuvant cisplatin and etoposside in patients with completely resected stage IIIIA small cell lung cancer: the Japan Clinical Oncology Lung Cancer Study Group Trial (JCOG9101). J Thorac Cardiovasc Surg 2005;129:977-83.

18. Norihisa Y, Nagata Y, Takayama K et al. Stereotactic body radiotherapy for oligometastatic lung tumors. Int $J$ Radiat Oncol Biol Phys 2008;72:398-403.

19. Shibamoto $\mathrm{Y}$, Otsuka S, Iwata $\mathrm{H}$ et al. Radiobiological evaluation of the radiation dose as used in high-precision radiotherapy: effect of prolonged delivery time and applicability of the linear-quadratic model. $J$ Radiat Res 2012;53:1-9.

20. Aupérin A, Arriagada R, Pignon JP et al. Prophylactic cranial irradiation for patients with small-cell lung cancer in complete remission. Prophylactic Cranial Irradiation Overview Collaborative Group. N Engl J Med 1999;341:476-84.

21. Le Péchoux CL, Dunant A, Senan S et al. Standard-dose versus higher-dose prophylactic cranial irradiation (PCI) in patients with limited-stage small-cell lung cancer in complete remission after chemotherapy and thoracic radiotherapy (PCI 99-01, EORTC 22003-08004,RTOG 0212, and IFCT 99-01): a randomised clinical trial. Lancet Oncol 2009;10: 467-74. 